\title{
Computer simulation of random packings for selfsimilar particle size distributions in soil and granular materials: porosity and pore size distribution
}

\author{
Miguel Angel Martín , Francisco J. Muñoz, Miguel Reyes \\ and F. Javier Taguas
}

\begin{abstract}
A 2-D computer simulation method of random packings is applied to sets of particles generated by a self-similar uniparametric model for particle size distributions in granular media. The parameter $p$ which controls the model is the proportion of mass of particles corresponding to the left half of the normalized size interval $[0,1]$. First the influence on the total porosity of the parameter $p$ is analysed and interpreted. It is shown that such parameter, and the fractal exponent of the associated power scaling, are efficient packing parameters, but this last one not in the way predicted in a former published work addressing an analogous research in artificial granular materials. The total porosity reaches the minimum value for $p=0.6$.

Limited information on the pore size distribution is obtained from the packing simulations and by means of morphological analysis methods. Results show that the range of pore sizes increases for decreasing values of $p$ showing also different shape in the volume pore size distribution. Further research including simulations with a greater number of particles and image resolution are required to obtain finer results on the hierarchical structure of pore space.
\end{abstract}


Keywords: soil, granular media, random packing, particle size distribution, self-similar mass-size distributions, fractals, power law scaling, porosity, morphological analysis, pore size distribution.

\section{Introduction}

Soil particle size distribution (PSD) is one of the most important physical attributes due to its great influence on soil properties. In fact in almost any pedotransfer function readily PSD available data, which are relatively easier to obtain, are used to estimate other soil parameters governing retention and transport of water and chemicals in soils (van Genuchten et al. ${ }^{1}$, Wösten et al. ${ }^{2}$, Pachepsky and Rawls ${ }^{3}$ )

Many attempts have been made to characterize soil particle distributions. In Buchan et al. ${ }^{4}$ five different models that were derived from the lognormal distribution function to describe soil PSD are compared.

Since the eighties Fractal Geometry has been used to describe scenarios with scale invariant features. Vaguely, the term fractal or self-similar is added to objects, distributions or processes when they have some scale invariant features and power laws revealing them. In such case the power exponents are usually called fractal dimensions.

The connection of fractals and fragmentation were firstly established in B Mandelbrot ${ }^{5}$. Later, in Turcotte $^{6}$ above seminal ideas were used to test the fractal scaling in soil thus establishing what nowadays is known as Turcotte law, namely, that the number $N(R)$ of particles greater than a characteristic diameter $R$ follows the scaling rule

$$
N(R) \approx R^{-D}
$$


being $D$ a number called the fragmentation fractal dimension.

Mass size distributions of particles have been described as power scaling of the type

$$
M(x<X) \approx X^{\alpha}
$$

for the cumulative number and mass distributions of soil particles of size $x$ less than a characteristic size $X$ (Perfect and $\mathrm{Kay}^{7}$, Perfect et $a l .{ }^{8}$, Rasiah et al. ${ }^{9}$ ). The relation between both exponents $\alpha=3-D$ may be established under standard assumptions in the soil sciences, such as constant density or spherical shape, usually considered for these transformations (Tyler and $\mathrm{Wheatcraft}^{10}$ ). Since then a large amount of papers have been devoted to test the fractal power law behavior of PSD as well as of different soil physical properties (see Anderson et al. ${ }^{11}$ for a review) which strongly support that fractal power law distributions might be helpful in order to describe soil properties. In Martin and Taguas ${ }^{12}$ a new way to parameterize and conceive the fractality of PSD, is presented. Although it is consistent with the fractal power law behavior it provides an especial mathematical formulation of the scale invariance of the mass-size distribution of soil particles that might be advantageous under some point of view (see Martin et $\left.a .^{13}\right)$

The PSD undoubtedly affects to soil porosity and to the hieratical geometry of pore space which are crucial to predict other physical properties as water retention or hydraulic conductivity. A first example of the effect of the heterogeneity of PSD on soil water retention arises from applications of the Arya and Paris ${ }^{14}$ method based on the hypothesis that soil water retention can be approximated by summing up water retention of pore subspaces related to the different soil particle sizes. In Tyler and Wheatcraft ${ }^{15}$ the tortuosity of pore channels is seen as a fractal dimension and related with the power scaling exponent of PSD. In Rieu and Sposito ${ }^{16}$ a fractal fragmentation model is 
presented for solid particles and pores and power scaling laws are derived from it for the hydraulic properties. A new approach to this problem was made by Perrier et al. ${ }^{17}$, Bird et al. ${ }^{18}$ and Perrier and Bird ${ }^{19}$ through the so called, "pore-solid fractal" model

The "invisible hand" which builds the bridge between PSD and pore space is the random packing arrangement of particles. The crucial influence of particle size distribution on the random packing and the corresponding pore structure increase the interest in relating both, either theoretically or by computational methods: it is an old dream among particulate scientist of different scientific fields. Even when the particles are modelled by hard spheres models of granular media addressing this issue may be useful in predicting different properties of a wide number of natural and engineering systems as soil, ceramics and porous materials. Sohn and Moreland ${ }^{20}$ studied the effect of Gaussian and lognormal distributions on packing density using dense random packing of sands, and Roualt and Assouling ${ }^{21}$ use a probabilistic approach to determine the distribution of the volume of the voids in packed spheres once their size distribution is given. Anishchik and Medvedev ${ }^{22}$ used a three-dimensional Apollonian packing as a model for dense granular systems investigating the particle size distribution and the fractal nature of packings. Packing of spheres with lognormal distributions by mean of computer simulation have been studied in Nolan and Kavanag ${ }^{23}$ and He et al. ${ }^{24}$ used a Monte Carlo simulation for a random model of spherical particles of sizes obeying any given distribution.

Andreasen and Andersen ${ }^{25}$ addressed together the modelling of PSD and the packing of particles of real granular media produced by grinding and involving particle sizes below a certain size. There, an original physicoempirical model with a mathematical formulation by means of a differential equation is proposed. Surprisingly the model postulate a power-law behaviour for the PSD of the type expressed in 
equation (2), the same that much later would be coined as "fractal". The exponent of the power-law scaling is there proposed as packing parameter. As far as the authors know, the proposal of these kind of original models for PSD (beyond the simple testing of classical distributions) providing simultaneously packing parameters, are scarce. This work is closely related with above pioneering work but incorporating new ingredients in the modelling and including computer simulations, adding also a particular interest in the soil scenario.

The goal of this paper is to use an efficient 2-D computational method "ad hoc" developed to simulate the random packing of self-similar fractal particle size distributions produced by the fractal PSD model of Martín and Taguas ${ }^{12}$. This model provides an exact self-similar PSD governed by a single input parameter driving the heterogeneity of the PSD. It produces a robust simulation of the PSD on the basis of such input that is not influenced by the random effect of any realization. All these features are important when the goal is finding clear relationships between self-similar PSD and the result of a random packing of particles. First the influence on the total porosity of the parameter defining the fractal model, is analyzed and interpreted. As a main result it is shown that such parameter, and the fractal exponent of the associated power scaling, are efficient packing parameters, but this last one not in the way predicted in Andreasen and Andersen ${ }^{25}$.

Finally the pore size distribution is obtained from the packing simulations and by means of morphological analysis methods established in Serra ${ }^{26}$. The relationship with the PSD is then studied. Since the self-similar model was tested with real soil data in Taguas et $a .^{27}$, the results here obtained might be useful in order to predict soil hydraulic properties using usual texture data as imputs. 


\section{Material and methods}

\subsection{The particle size distribution model}

In practical terms the statistical similarity property for a grain distribution might be defined by the requirement that after sieving some amount of granular material with sieves of different size, the structure of the grain distribution of the material sieved is equivalent (in information terms) to the grain distribution of the initial one. This practical way of understanding scale invariance lead to a self-similar mass-size distribution as a model of PSD which is consistent with a power law scaling (see Martín et $\left.a l .{ }^{13}\right)$.

Let us suppose that the granular material is sieved retaining grains of size greater than $r$ (think $r=1 / 2$ ). Suppose that the fraction of material sieved is $p_{1}=p$ and the retained part $p_{2}=1-p$.

Let $w_{1}$ and $w_{2}$ be the linear transformations $w_{1}(x)=x / 2$ and $w_{2}(x)=(x+1) / 2$ be the linear transformations which transform the interval $[0,1]$ into the subintervals $[0,1 / 2]$ and $[1 / 2,1]$, respectively.

Following Martín and Taguas ${ }^{12}$, the invariant measure $\boldsymbol{\mu}$ verifying

$$
\mu(I)=\sum_{i=1}^{2} p_{i} w_{i}^{-1}(I)
$$

is proposed as a model for PSD. Because of self-similarity the parameter $p$ drives the heterogeneity of the PSD establishing the relative predominance of smaller particles respect to the greater ones in consecutive size classes across scales.

The measure of any subinterval $I$, as well as the corresponding accumulated distribution function

$$
\mu(x)=\int_{0}^{x} d \mu(x)
$$

can be easily estimated using Elton's theorem (see Martín and Taguas ${ }^{12}$ ). 
The mass-size distribution $\mu$ is of multifractal type which means that it presents a continuum of different mass accumulation degrees (Hölder exponents) which produces a "curdling" effect for the accumulated distribution function (see Feder ${ }^{28}$ ). As a result this accumulated distribution can not be described by elemental mathematical formulas, except if $p=1 / 2$ (in that case $\mu$ is the uniform distribution)

In spite of such multifractal nature, however, self-similar measures present a regularity in the average value of the Hólder exponents within (small) subintervals (see Everstz and Mandelbrot ${ }^{29}$ ), which allows to expect that the simulated accumulated distribution fits statistically to a power-law "smooth" model as equation (2). In fact, both models, any of them with particular features, aim to describe the scale invariance of the PSD with mathematical tools of quite different nature (see Martín et $a l .^{13}$ )

\subsection{Computer simulation of packing}

A Monte Carlo-based method following the model proposed in Vidal et al. ${ }^{30}$ has been considered for the generation of packing processes. This method randomly positions a sequence of particles, ordered by decreasing radius, in a square domain with periodic border and edge length $L_{0}$. The center of each particle, with randomly generated coordinates $(x, y)$, will always remain in the domain as long as it does not overlap any previously positioned particle. There exist a maximum number of attempts (of randomly generated $(x, y)$ ), $N_{T M}$, of the positioning of each particle within the domain. There also exists a number, $N_{R}$, of the repetition of the positioning of the same sequence of particles within the domain. Once all the particles have been positioned, a new domain will be defined with the length of the smaller edge, $L_{1}$, where $L_{1}$ is defined as of $L_{1}=k \cdot L_{0}$, with $0<k<1$ being a reduction factor of the domain. This positioning process will continue repeating in the new domain $L_{i}$, so that $L_{i}=k \cdot L_{i-1}$, 
until stage $n$ at which a new particle will exist whose number of positioning attempts reaches $N_{T M}$. At this point the result of the packing simulation of the repetition of the completed $N_{R}$ will be that obtained at stage $n-1$ where all the particles have been positioned within the domain of size $L_{n-1}$. The void fraction, $V$, resulting from that packing will be

$$
V=1-\frac{S_{T}}{L_{n-1}^{2}}
$$

where $S_{T}$ is the total surface area of all the particles of the sequence.

For the execution of the packing simulations, the initial size of the domain, $L_{0}$, has been obtained as a function of the total surface area of the particles by means of the equation $L_{0}=\left(S_{T} \cdot 2,5\right)^{1 / 2}$ which produces an initial void fraction $V=60 \%$. The reduction factor $k$ has been fixed at 0.999 and the maximum number of iterations at $10^{6}$. Each particle sequence repeats $N_{R}=10$ times and the value of the ending void fraction $V$ of the simulation for that distribution will be the lowest of these repetitions.

\subsection{The pore size distribution}

The pore size distribution is obtained by morphological analysis of the images obtained in each simulation. Figure 1 (A, B, C, D) shows an example of how the morphological granulometry method (also called morphological sieving) works on the pores of an image of a granular medium. 

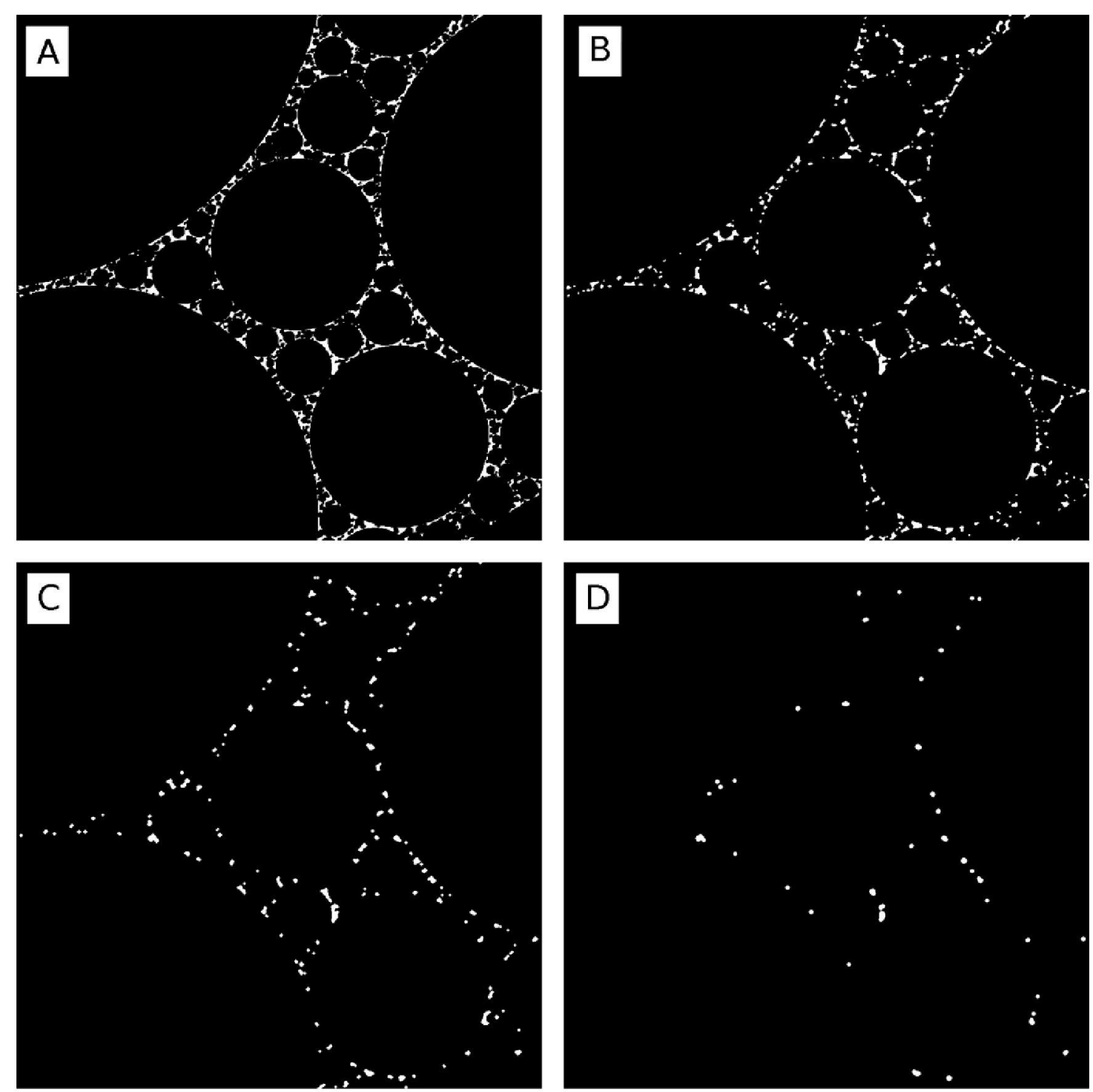

Figure 1. Example of how morphological granulometry method works on the pores (represented in white) of an image corresponding to a PSD obtained with $p=0.6$. Figures A, B, C and D are different stages of the process where smaller pores of the previous step have disappeared.

At different stages of the process, the pores of smaller size (smaller than a certain radius $\delta$ ) are disappearing, allowing us to measure the volume (or area in 2D case) of the remaining pores. This method is based on the two basic operations of mathematical morphology $\left(\operatorname{Serra}^{26}\right)$, erosion and dilation and the criterion of pore size used in the method reflects the idea of hydraulic radius, i.e, the shape of the meniscus of water in every part of the pore space (Vogel and Roth ${ }^{31}$ ). The consecutive application of the two basic morphological operations: erosion, that produces a reduction or shrinking of size $\delta$ of the pores, followed by a dilation, that produces a growing of the resulting region 
makes that the pores smaller than $\delta$ disappear during the erosion and not recover their size during the dilation as it happens with the other pores that preserving its original sizes. The difference between the initial stage and the current stage results in the total volume of pores with size less than $\delta$. The successive application of this procedure, with radius $\delta$ increasing allows us to obtain the curve that defines the pore size distribution of a granular medium. A more detailed description of this procedure can be found in Ohser and Mucklich ${ }^{32}$.

\section{Results and discussion}

Following the PSD model, a number of 9 self-similar mass-size distributions have been generated using the values $p=0.1, p=0.2, \ldots, p=0.9$. Each mass-size distribution is supposed formed by 2-D spherical particles. Assuming constant density a number-size distribution is obtained. The probability of appearance of a particle of size

$i$ in each simulation is $\frac{n_{i}}{\sum n_{i}}$ being $n_{i}$ the number of particles of size $i$ in each number-size distribution. Any simulation is considered a sample which represents the granular medium. A number of 10 different realizations of the packing algorithm are implemented in order to generate mean values.

Figure 2 shows an example (and its magnification) of the packing result obtained with a PSD with $p=0.6$. 


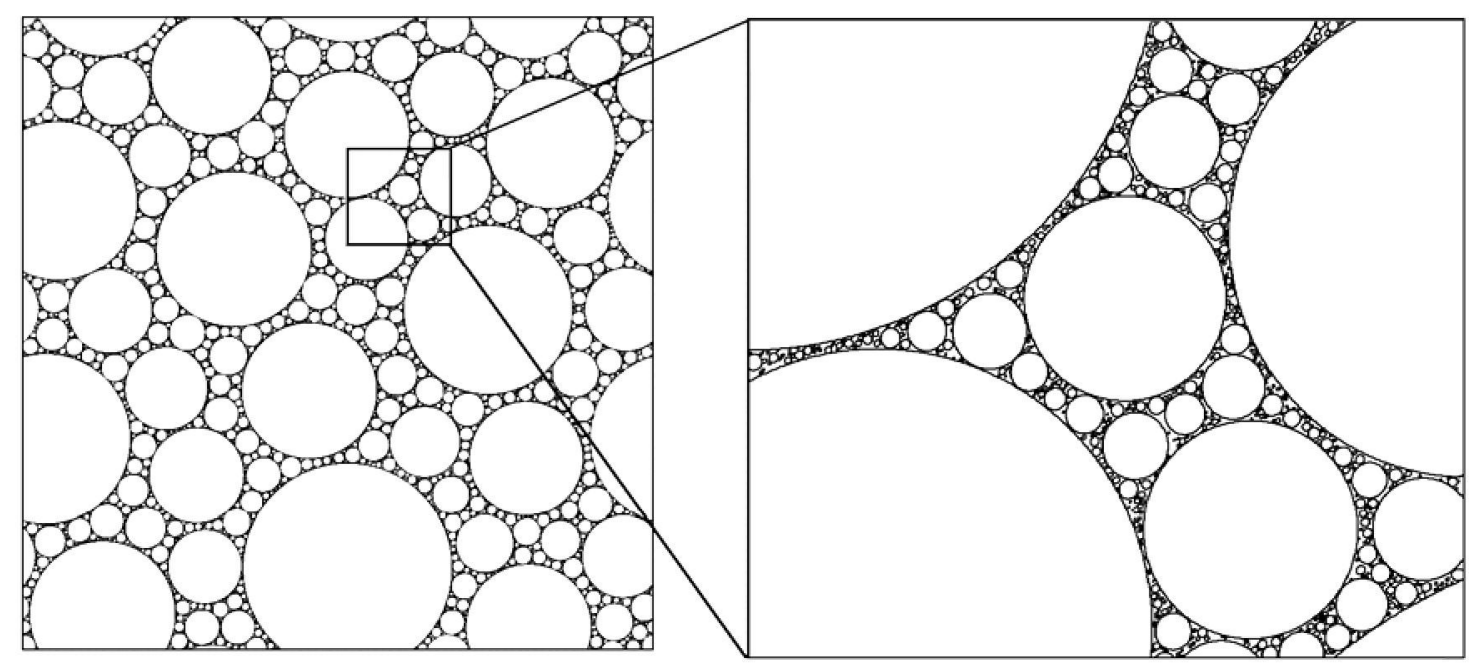

Figure 2. An example and its magnification of the result of the packing algorithm corresponding to a PSD obtained with $p=0.6$.

Figure 3 shows the variance of the computed porosity against the size of the number of particles used in the simulation for different values of $p$. It is clear that the oscillation of the porosity decreases and approaches to zero at a threshold which depends on $p$. The number of particles used in the simulations is always a value greater than such thresholds: 10.000 particles for $p=0.1,0.2$ and 0.9 , and 50.000 particles for the rest of $p$ values. This supports the reliability of the simulations made and the later analysis.

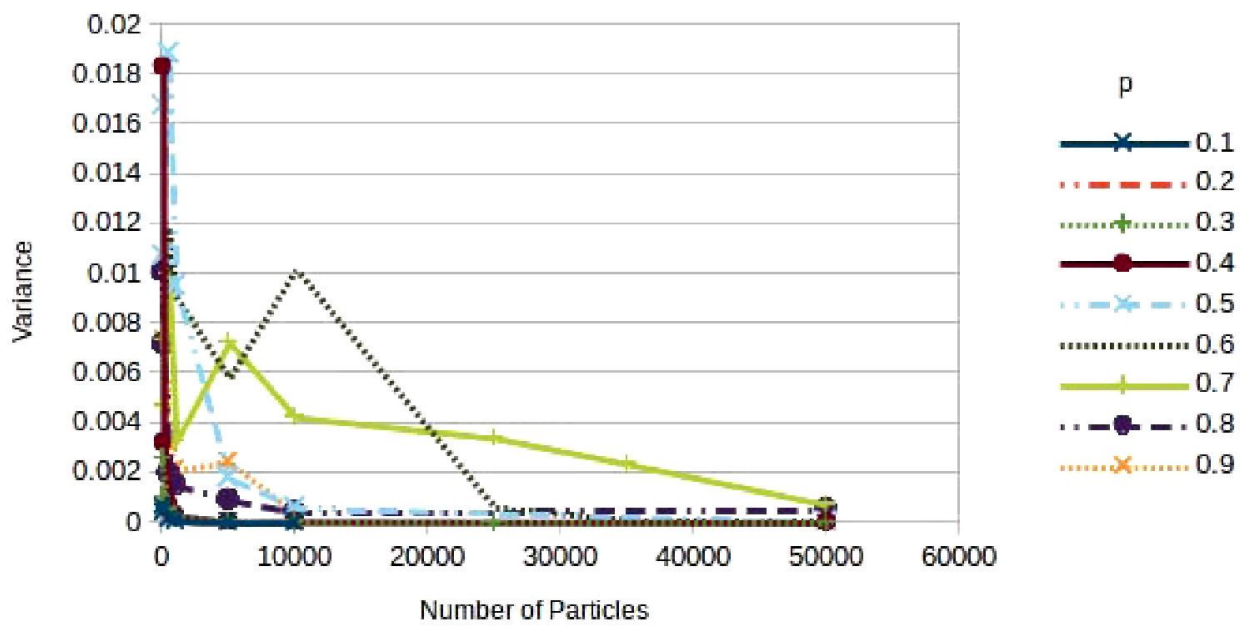

Figure 3. Variance of the computed porosity against of the number of particles used for different values of $p$. 
Figure 4 shows the mean value of the porosity obtained with 10 different realizations of the packing algorithm for any value of $p$.

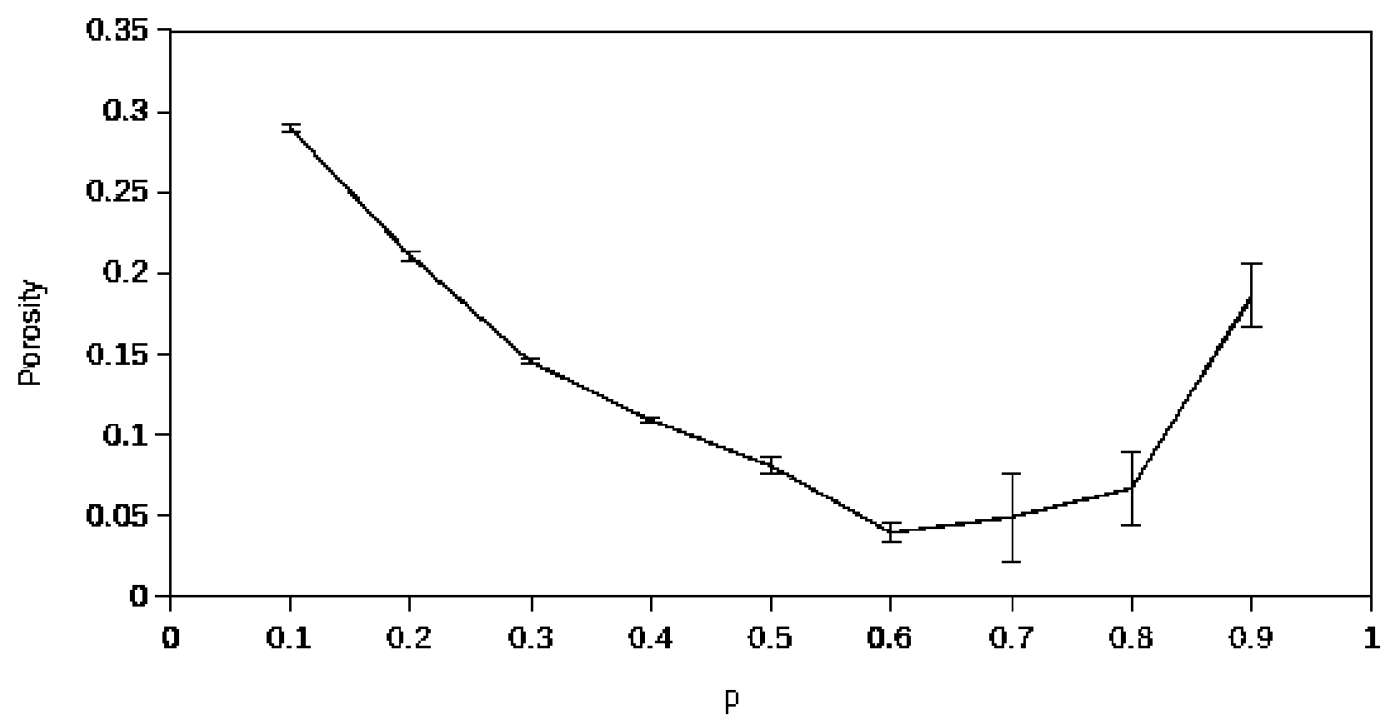

Figure 4. Mean value of porosity obtained for $p=0.1$ to $p=0.9$. The error bars indicate the standard deviations.

A first important result is that the total porosity has a clear dependence of the value $p$ which reflects the relative predominance of the lower grain sizes in relation with the greater ones (at every scale because of self-similarity). This might be because the intergranular space between grains of a certain size class is occupied by grains of a correlatively lower size class: the degree of this occupation would be affected by the mentioned predominance.

Table 1 establishes the relation between the parameter $p$ and the power scaling exponent $\alpha$ of the accumulated mass-size distribution. High $R^{2}$ values of the corresponding log-log plot fitting can be observed, as expected. It can observed, however, that those $R^{2}$ values are smaller for extreme values of $p(p=0.1$ and $p=$ 0.9). This have a rather consistent explanation: for those values of $p$ the heterogeneity, and the "curdling" effect is higher and because of that a worse fitting is expected. Also 
the reliability of the $\alpha$ values should be expected smaller. As a matter of fact values of $\alpha$ greater than 3 should be understood that have a numerical/computational origin.

\begin{tabular}{|c|c|c|}
\hline $\mathbf{p}$ & $\boldsymbol{\alpha}$ & $\mathbf{R}^{2}$ \\
\hline 0.1 & 3.065 & 0.974 \\
\hline 0.2 & 2.194 & 0.987 \\
\hline 0.3 & 1.675 & 0.995 \\
\hline 0.4 & 1.299 & 0.999 \\
\hline 0.5 & 1.000 & 1.000 \\
\hline 0.6 & 0.750 & 0.999 \\
\hline 0.7 & 0.532 & 0.994 \\
\hline 0.8 & 0.338 & 0.986 \\
\hline 0.9 & 0.162 & 0.972 \\
\hline
\end{tabular}

Table 1. Relationship between parameter $p$ and power scaling exponent $\alpha$ and its respective correlation coefficient.
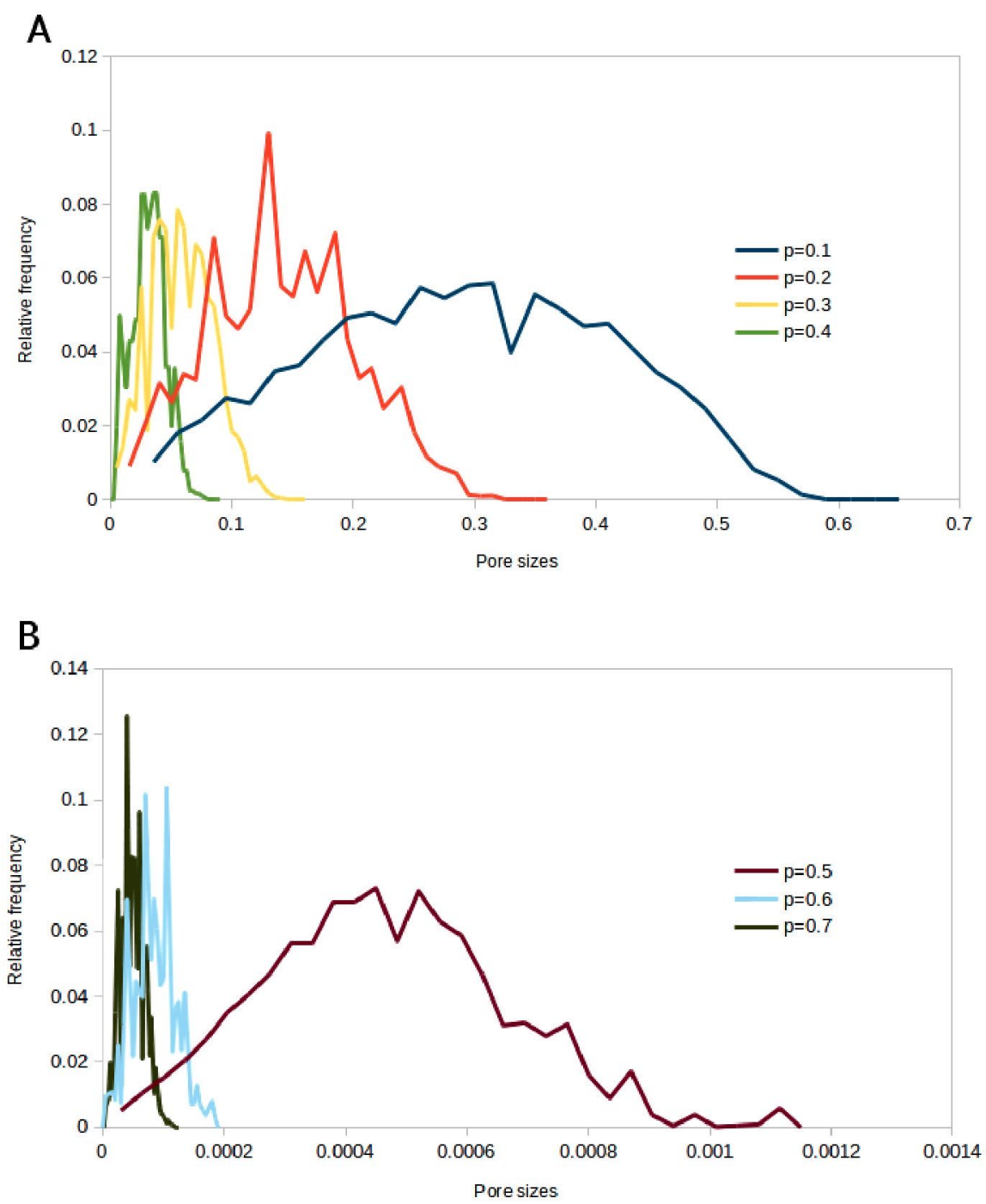
Figure 5. Pore volume size distributions measured using image morphological analysis. In (A) obtained from packing simulations with $p=0.1$ to $p=0.4$. The interval of sizes $[0,1]$ corresponds to $[0,100$ pixels]. In (B) obtained from packing simulations with $p=0.5$ to $p=0.7$. The interval of sizes $[0,1]$ corresponds to $[0,100.000$ pixels $]$.

It can be observed that the porosity does not present a monotonically increasing behaviour as a function of $\alpha$ as was predicted in Andreasen and Andersen ${ }^{25}$. As a matter of fact it takes a minimum value for $\alpha=0.750$. This has a quite clear explanation: the occupation of the intergranular space should take an optimal degree for a certain value of the above mentioned predominance of the lower grain sizes with respect to the greater ones. Around this optimum point, which should be close to the Apolonian packing used as a model of dense granular media (see Anishchik and Medvedev ${ }^{22}$ ), random packings are expected to move away of that optimum scenario and to have a greater intergranular space, thus agreeing with the results here presented.

Figure 5 shows pore size distribution density for different values of $p$. Because of size limitations in the digital image used in the morphological analysis, it is necessary to change image resolution for the different ranges of $p$ values in order to capture the representative hieratical structure of pore space: for small values of $p$ there are a greater relative abundance of big particles and a low resolution is needed. Similarly bigger values of $p$ require the use of higher resolution.

The same trend may be observed for the different range of $p$. Results in figure $5 \mathrm{~A}$ and $5 \mathrm{~B}$ show in both cases that the range of pore sizes increases for decreasing values of $p$ showing also different shape in the volume pore size distribution. Results seem to be consistent with the meaning of the parameter $p$ : on one hand for small values of $p$ the probability of greater particles increases and also increases the chance of bigger pores. On the other the diversity of particle sizes should favour the filling of intergranular space. Further research including simulations with a greater number of particles and 
image resolution are required to obtain finer results on the hierarchical structure of pore space.

\section{Conclusions}

The fractal PSD model of Martín and Taguas ${ }^{12}$ providing an exact self-similar PSD governed by a single input parameter $p$ is suitable for finding relationships between self-similar PSD and the value of the porosity of simulated random packing of particles.

Numerical analysis shows that the 2-D computational method "ad hoc" developed to simulate the random packing of particles is efficient and reliable.

It is shown that the parameter $p$, and the fractal exponent $\alpha$ of the associated power scaling, are efficient packing parameters, but this last one not in the way predicted in Andreasen and Andersen ${ }^{25}$. The porosity reaches the minimum value for $p=0.6$

Also it is shown that the range of pore size increases for decreasing values of $p$ showing also different shape in the volume pore size distribution.

\section{Acknowledgment:}

This research work was been funded by Spain's Plan Nacional de Investigación Científica, Desarrollo e Innovación Tecnológica (I+D+I) under ref. AGL2011-25175.

\section{References}

1. M. Th. van Genuchten, F. J. Leij and L. J. Lund (eds.), Indirect Methods of Estimating the Hydraulic Properties of Unsaturated Soils, (Proc. Int. Workshop, Univ. of California, Riverside, 1992) 
2. J. H. M. Wösten, Y. A. Pachepsky and W. J. Rawls, Pedotransfer functions: bridging the gap between available basic soil data and missing soil hydraulic characteristics, J. Hydrol. 251 (3-4) (2001) 123-150.

3. Y. A. Pachepsky and W. J. Rawls, Development of Pedotransfer Functions in Soil Hydrology, (Elsevier, Amsterdam, 2004).

4. G. D. Buchan, K. S. Grewal and A. B. Robson, Improved models of particle-size distribution: an illustration of model comparison techniques, Soil Sci. Soc. Am. J. 57 (1993) 901-908.

5. B. B. Mandelbrot, The fractal geometry of nature, (W.H. Freeman, New York, 1982).

6. D. L. Turcotte, Fractals and fragmentation, J. Geophys. Rev. 91 (1986) 1921-1926.

7. E. Perfect and B. D. Kay, Fractal theory applied to soil aggregation, Soil Sci. Soc. Am. J. 55 (1991) 1552-1558.

8. E. Perfect, V. Rasiah and B. D. Kay, Fractal dimensions of soil aggregate-size distributions calculated by number and mass, Soil Sci. Soc. Am. J. 56 (1992) 14071409 .

9. V. Rasiah, B. D. Kay and E. Perfect, Evaluation of selected factors influencing aggregate fragmentation using fractal theory, Canadian J. Soil Sci. 72 (1992) 97106.

10. W. S. Tyler and S. W. Wheatcraft, Fractal scaling of soil particle-size distributions: analysis and limitations, Soil Sci. Soc. Am. J. 56 (1992) 362-369.

11. A. N. Anderson, A. D. McBratney and J. W. Crawford, Applications of fractals to soil studies, Advances in Agron. 63 (1998) 1-76.

12. M. A. Martín and F. J. Taguas, Fractal modelling, characterization and simulation of particle-size distributions in soil, Proc. R. Soc. Lond. A 454 (1998) 1457-1468. 
13. M. A. Martin, M. Reyes and F. J. Taguas, On the Generative Equations of Fractal Self-Similarity in Granular Media and the Related PSD Models, Vadose Zone J. 12(3) (2013) doi:10.2136/vzj2012.0207.

14. L. M. Arya and J. F. Paris, A physicoempirical model to predict soil moisture characteristics from particle-size distribution and bulk density data, Soil Sci. Soc. Am. J. 45 (1981) 1023-1030.

15. W. S. Tyler and S. W. Wheatcraft, Application of fractal mathematics to soil water retention, Soil Sci. Soc. Am. J. 53 (1989) 987-996.

16. M. Rieu and G. Sposito, Fractal fragmentation, soil porosity and soil water properties: I. Theory, Soil Sci. Soc. Am. J. 55 (1991) 1231-1238.

17. E. Perrier, N. Bird and M. Rieu, Generalizing the fractal model of soil structure: the pore-solid fractal approach, Geoderma 88 (1999) 137-164.

18. N. Bird, E. Perrier and M. Rieu, The water retention curve for a model of soil structure with pore and solid fractal distributions, Eur. J. Soil Sci. 55 (2000) 55-63.

19. E. Perrier and N. Bird, Modeling soil fragmentation: the pore solid fractal approach, Soil Tillage Res. 64 (2002) 91-99.

20. H.Y. Sohn and C. Moreland, The effect of particle size distribution on packing density, Can. J. Chem. Eng. 46 (1968) 162-167.

21. Y. Roualt and S. Assouline, A probabilistic approach towards modeling the relationships between particle and pore size distributions: the multicomponent packed sphere case, Powder Technol. 96 (1998) 33-41.

22. S.V. Anishchik and N.N. Medvedev, Three-Dimensional Apollonian Packing as a Model for Dense Granular Systems, Phys. Rev. Lett. 75 (1995) 4314-4317.

23. G.T. Nolan and P.E. Kavanag, Computer simulation of random packings of spheres with log-normal distributions, Powder Technol. 76 (1993) 309-316. 
24. D. He, N.N. Ekere and L. Cai, Computer simulation of random packing of unequal particles, Phys. Rev. E60 (1999) 7098-7104.

25. A. Andreasen and J. Andersen, Ueber die Beziehung zwischen Kornabstufung und Zwischenraum in Produkten aus losen Ko“rnern, Kolloid-Zeitschrift 50 (1930) 217228.

26. J. Serra, Image analysis and mathematical morphology, (Academic Press, New York, 1983).

27. F. J. Taguas, M. A. Martín and E. Perfect, Simulation and testing of self-similar structures for soil particle-size distributions using iterated function systems, Geoderma 88 (1999) 191-203.

28. J. Feder, Fractals, (Plenum Press, New York, 1988).

29. C.J.G. Evertz and B.B. Mandelbrot, Multifractal measures, In Chaos and Fractals, eds. H.O. Peitgen et al. (Springer Verlag, New York, 1992), 921-953.

30. D. Vidal, C. Ridgway, G. Pianet, J. Schoelkopf, R. Roy and F. Bertrand, Effect of particle size distribution and packing compression on fluid permeability as predicted by lattice-Boltzmann simulations, Computers and Chemical Engineering 33 (2009) 256-266.

31. H. J. Vogel and K. Roth, Quantitative morphology and network representation of soil pore structure, Adv. Water Res. 24 (2001) 233-242.

32. J. Ohser and F. Mücklich, Statistical Analysis of Microstructures in Materials Science, (John Wiley \& Sons, Chichester, 2000). 